\title{
Aligned magnetic field on dusty Casson fluid over a stretching sheet with Newtonian heating
}

\author{
Nur Syamilah Arifin a, Syazwani Mohd Zokri a, Abdul Rahman Mohd Kasim a, ${ }^{*}$, Mohd Zuki Salleh a, \\ Wan Nur Syahidah Wan Yusoff a, Nurul Farahain Mohammad ${ }^{b}$, Sharidan Shafie ${ }^{c}$ \\ a Applied \& Industrial Mathematics Research Group, Faculty of Industrial Sciences \& Technology, Universiti Malaysia Pahang, 26300 UMP \\ Kuantan, Pahang, Malaysia \\ b Department of Computational and Theoretical Sciences, Kulliyyah of Sciences, International Islamic University Malaysia, 25200 Kuantan, Pahang, \\ Malaysia \\ c Department of Mathematical Sciences, Faculty of Science, Universiti Teknologi Malaysia, 81310 UTM Johor Bahru, Johor, Malaysia \\ * Corresponding author: rahmanmohd@ump.edu.my
}

\section{Article history}

Received 18 February 2017

Accepted 4 July 2017

\begin{abstract}
Boundary layer flow and heat transfer on Casson fluid with dust particle over a stretching sheet is numerically investigated. The influences of aligned magnetic field together with Newtonian heating are considered in this problem. The governing equations are first transformed into ordinary differential equations using the appropriate similarity transformation variables. The numerical computation using Runge-Kutta Fehlberg (RKF45) method is employed to generate the results. Several physical parameters for both phases (fluid and particle) such as aligned angle, magnetic field parameter, Casson parameter, fluid particle interaction parameter, Prandtl number and conjugate parameter are investigated and analysed. The results in term of distribution velocity and temperature are presented graphically. The finding revealed that a rise in aligned angle and magnetic field parameter led to decrease the velocity profile and increase the temperature profile for both phases.
\end{abstract}

Keywords: Aligned magnetic field, two-phase flow, Casson fluid, stretching sheet, Newtonian Heating

\section{INTRODUCTION}

The multiphase flow is the interaction of several phase (solid, liquid and gas). The flow of solid-liquid is one of the simplest multiphase flow which is normally encountered in the flow of mud, flow of corpuscles in plasma and sedimentation. The two-phase flow is studied in such a way that the governing equations is separate accordingly. The propulsion and combustion in rockets and transport of powder materials suspension in pipe are some of engineering applications of fluid flow with particle suspension (Datta and Mishra, 1982). Gopinath, 2008 revealed the flow in magnetohydodynamic (MHD) generator is affected by the aggregation of slag and seeded particle in the channel.

The growth of particle such as ash or soot on the wall of certain engineering machines have led to a reforming the interest in the study of fluid flow embeded with particle suspension. The concentration of dust and a relaxation time of dust particle are the major influence of dust particle on gases which has pionered discussed by (Saffman, 1962). The boundary layer theory of dusty gas past an infinite plate was investigated by (Chakrabarti, 1974). The hydrodyamic of dusty fluid flow over a stretching with heat transfer and variable fluid properties effect was carried out by (Vajravelu et al., 2013). In additional to the work of (Ramesh et al., 2015) on the convective surface of dusty fluid over a stretching sheet, (Isa et al., 2016) has taking challenges to study the stagnation point flow by considering the magnetic field effect towards the fluid. Meanwhile, the mixture of water and oil with metal particles on the natural convection flow over a heated vertical surface has been conducted by (Siddiqa et al., 2015).
Recent development on the industrial applications have heightened the need for non-Newtonian fluid due to some restriction in properties of Newtonian fluid. Processing of plastic foam and food stuft, production of plastic sheets and extrusion of molten polymers are several applications of non-Newtonian fluid flow. A number of studies have attempted to develop the non-Newtonian fluid model with various of physical effect (Aurangzaib et al., 2013). One of the non-Newtonian fluid model that possess the interactive behaviour of solid and liquid phases is recognized as Casson fluid. The human blood contained protein, fibrinogen and globulin in an aqueous base plasma can be clasify as Casson fluid (Mukhopadhyay et al., 2013). A more recent analysis in the influence of aligned magnetic field on the boundary layer flow of Casson fluid and viscous fluid over a stretching sheet has been highlighted as in (Hakeem et al., 2016; Kalaivanan et al., 2015; Nur Syamilah et al., 2016). In another study, (Sandeep et al., 2015) examined the mixture of conducting dust particle in a nanofluids (dusty nanofluid) with the present of magnetic field over a permeable stretching/shrinking surface.

The fluid filled with the dust particle has been one of the major interesting research subject due to the various applications, therefore this paper attempts to provide a more detailed investigation regarding the effects of dust particle in a Casson fluid flow over a stretching sheet. The aligned magnetic field with thermal boundary condition, Newtonian heating $(\mathrm{NH})$ is also considered. The governing equations of two-phase model are first transformed into ordinary differential equations using a similarity transformation before numerical computation can be carried out . The influence of several physical 
parameters on velocity as well as temperature of fluid are analysed through table and graph.

\section{PROBLEM FORMULATION}

The steady, incompressible two dimensional boundary layer flow of dusty Casson fluid over a stretching sheet with a stretching linear velocity $u_{w}(x)=a x$ is considered. An aligned magnetic field with an acute angle $\alpha_{1}$ is applied to the flow as illustrates in Figure 1. The assumptions where dust particles are in spherical shape, uniform size and number density are taken as constant throughout the flow is deliberated.

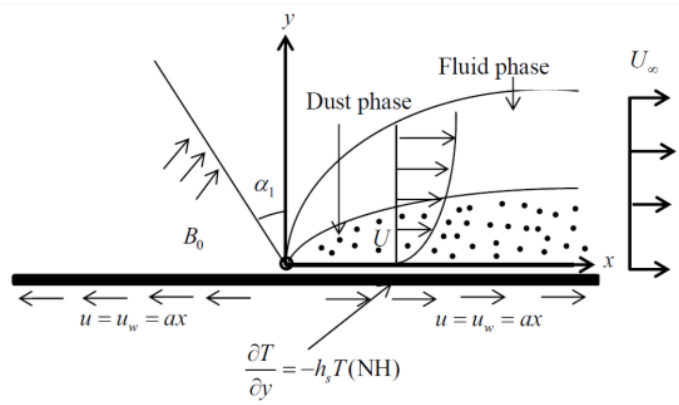

Fig. 1 Flow configuration.

Under the boundary layer and Boussinesq approximations, the momentum and energy equations for two-phase flow can be written as:

Fluid phase:

$\frac{\partial u}{\partial x}+\frac{\partial v}{\partial y}=0$

$$
\rho\left(u \frac{\partial u}{\partial x}+v \frac{\partial u}{\partial y}\right)=\mu\left(1+\frac{1}{A}\right)\left(\frac{\partial^{2} u}{\partial y^{2}}\right)+\frac{\rho_{p}}{\tau_{v}}\left(u_{p}-u\right)-\sigma u B_{0}^{2} \sin ^{2} \alpha_{1},
$$

$\rho c_{p}\left(u \frac{\partial T}{\partial x}+v \frac{\partial T}{\partial y}\right)=k\left(\frac{\partial^{2} T}{\partial y^{2}}\right)+\frac{\rho_{p} c_{s}}{\gamma_{T}}\left(T_{p}-T\right)$

Dust phase:

$\frac{\partial}{\partial x}\left(u_{p}\right)+\frac{\partial}{\partial y}\left(v_{p}\right)=0$

$\rho_{p}\left(u_{p} \frac{\partial u_{p}}{\partial x}+v_{p} \frac{\partial u_{p}}{\partial y}\right)=\frac{\rho_{p}}{\tau_{v}}\left(u-u_{p}\right)$

$\rho_{p} c_{s}\left(u_{p} \frac{\partial T_{p}}{\partial x}+v_{p} \frac{\partial T_{p}}{\partial y}\right)=-\frac{\rho_{p} c_{s}}{\gamma_{T}}\left(T_{p}-T\right)$

where $(u, v)$ and $\left(u_{p}, v_{p}\right)$ are the velocities components of the fluid and particle phase along $x$ and $y$ axes, respectively. $\mu$ is the coefficient of viscosity of the fluid, $\rho$ and $\rho_{p}$ are the density of fluid and dust phase, $\alpha_{1}$ is the aligned angle, $\tau_{v}=1 / k$ is the relaxation time of particles phase, $k$ is the Stoke's resistance (drag force), $c_{p}$ and $c_{s}$ are specific heat of fluid and dust particle, $T$ and $T_{p}$ are the temperature of fluid and particle phase, $\gamma_{T}$ is the thermal relaxation time, $B_{0}$ is the magnetic-field strength, $A=\mu_{B} \sqrt{2 \pi_{c}} / p_{y}$ is the nonNewtonian (Casson) parameter and $h_{s}$ is heat transfer parameter.

Here, $a$ is the positive constant and the boundary conditions for the present problem are

$u=u_{w}(x)=a x, v=0, \quad \frac{\partial T}{\partial y}=-h_{s} T \quad$ at $y=0$, $u \rightarrow 0, u_{p} \rightarrow 0, v_{p} \rightarrow v, T \rightarrow T_{\infty}, T_{p} \rightarrow T_{\infty} \quad$ as $y \rightarrow 0$

The similarity transformations (8) have been introduced to the governing equations (1) to (6) to transform them into dimensionless equations,

$u=a x f^{\prime}(\eta), \quad v=-\sqrt{a v} f(\eta), \quad \eta=\sqrt{\frac{a}{v}} y, \quad \theta(\eta)=\frac{T-T_{\infty}}{T_{\infty}}$

$u_{p}=a x F^{\prime}(\eta), v_{p}=-\sqrt{a v} F(\eta), \quad \theta_{p}(\eta)=\frac{T_{p}-T_{\infty}}{T_{\infty}}$,

where $\psi$ is the stream function defined as $u=\frac{\partial \psi}{\partial y}$ and $v=-\frac{\partial \psi}{\partial x}$. Now, the transformed ordinary differential equations (1)-(6) can be expressed as

$\left(1+\frac{1}{A}\right) f^{\prime \prime \prime}(\eta)+f(\eta) f^{\prime \prime}(\eta)-\left(f^{\prime}(\eta)\right)^{2}+\beta N\left(F^{\prime}(\eta)-f^{\prime}(\eta)\right)$

$-M \sin ^{2} \alpha_{1} f^{\prime}(\eta)=0$,

(9)

$$
\begin{aligned}
& \theta^{\prime \prime}(\eta)+\operatorname{Pr} f(\eta) \theta^{\prime}(\eta)+\frac{2}{3} \beta N\left(\theta_{p}(\eta)-\theta(\eta)\right)=0, \\
& \left.\left(F^{\prime}(\eta)\right)^{2}-F(\eta) F^{\prime \prime}(\eta)+\beta\left(F^{\prime}(\eta)-f^{\prime}(\eta)\right)\right]=0, \\
& \theta_{p}{ }^{\prime}(\eta) F(\eta)+\frac{2}{3} \frac{\beta}{\operatorname{Pr} \lambda}\left(\theta(\eta)-\theta_{p}(\eta)\right)=0
\end{aligned}
$$

and boundary conditions $(7)$ reduces to

$f(0)=0, f^{\prime}(0)=1, \quad \theta^{\prime}(0)=-\gamma(1+\theta(0))$ as $\eta=0$

$f^{\prime}(\eta) \rightarrow 0, F(\eta) \rightarrow 0, F(\eta) \rightarrow f(\eta), \theta(\eta) \rightarrow 0, \theta_{p}(\eta) \rightarrow 0$ as $\eta \rightarrow \infty$

where a prime denotes differentiation with respect to $\eta . \quad N=\rho_{p} / \rho$ is the mass concentration of particle phase, $M=\sigma B_{0}^{2} / \rho a$ is the magnetic field parameter, $\beta=1 / a \tau_{v}$ is the fluid-particle interaction parameter, $\operatorname{Pr}=\mu c_{p} / k$ is the Prandtl number, $\lambda=c_{s} / c_{p}$ is the specific heat ratio of mixture and $\gamma=-h_{S}(v / a)^{1 / 2}$ is the conjugate parameter for $\mathrm{NH}$.

According to (Andersson et al., 1992), the exact solution for Eq. (9) by considering only magnetic field is expressed as

$f(\eta)=\frac{1}{m}\left(1-e^{-m \eta}\right)$

where $m=\sqrt{1+M^{2}}$. Using (14), then the exact expression for temperature profile is

$\theta(\eta)=C_{1} \int_{\eta}^{\infty} e^{-\operatorname{Pr} \int_{\eta}^{\infty} f d \eta} d \eta$

where $C_{1}=\frac{\gamma(1-\theta(0))}{e^{-\operatorname{Pr} \int_{\eta}^{\infty} f d \eta}}$. The non-dimensional quantities of physical interest of the the skin friction coefficient $C_{f}$ and the local Nusselt number $N u_{x}$ are defined by

$C_{f} \operatorname{Re}_{x}^{1 / 2}=f^{\prime \prime}(0), N u_{x} \operatorname{Re}_{x}^{-1 / 2}=\gamma\left(1+\frac{1}{\theta(0)}\right)$,

where $\operatorname{Re}_{x}=\left(a x^{2} / v\right)$ is the Reynolds number.

\section{NUMERICAL PROCEDURE}

The analytical solutions is difficult to obtained for the complexity of the present governing equations. For that, the numerical solution of Runge-Kutta Fehlberg (RKF45) method on Maple software is employed to solve the system of ordinary equations (9) - (12) with boundary conditions (13) in order to evaluate the flow properties and 
heat transfer for both fluid and dust phases. The maximum finite boundary layer thickness $\eta_{\infty}=5$ and 8 is computed to satisfy the boundary conditions asymptotically for both phases.

\section{RESULTS AND DISCUSSION}

The governing equations of dusty Casson fluid model comprises of two part which are fluid and dust phases. The flow and heat transfer of the present problem are analysed graphically through several physical parameters, namely, particle-fluid interaction parameter $\beta$, Casson parameter $A$, aligned angle $\alpha_{1}$, magnetic field parameter $M$, Prandtl number $\operatorname{Pr}$ and conjugate parameter, $\gamma$. The values of skin friction coefficeint and Nusselt number are also tabulated in Table 3. To determine the correctness of the present results, comparison of skin friction coefficient $f^{\prime \prime}(0)$ and surface temperature $\theta(0)$ with existed work are made for some limitating cases in the absence of fluid particle interaction parameter and Casson parameter (see Table 1 and 2). It was found, the analytic solutions of (Andersson et al., 1992) and Eq. (15) as well as numerical solution presented in (Salleh et al., 2010) was consistent with the present findings. The numerical results of velocity $\left(f^{\prime}(\eta), F^{\prime}(\eta)\right)$ and temperature profiles $\left(\theta(\eta), \theta_{p}(\eta)\right)$ for fluid and dust phases, respectively are computed for several fixed pertinent parameter which

are $\alpha_{1}=\pi / 6, M=2, \beta=1, \gamma=0.5, A=1, N=12.6, \operatorname{Pr}=16.7$ and $\lambda=0.25$

Fig. 2 and Fig. 3 show the effect of Casson parameter, $A$, for the fluid flow with and without dust particle. It should be noted that, the present problem turns to the those classical problem of single phase flow at $\beta=0$. From Fig. 2, the decreasing pattern of fluid velocity is observed with increasing on values of $A$, for both Casson $(\beta=0)$ and dusty Casson fluid $(\beta=1)$. Also, the fluid velocity is promptly achieve the asymptotic behaviour when $\beta=1$ compared to $\beta=0$. It is worth to mention that the flow of fluid tend to decelerate in the present of dust particles. In Fig. 3, the decreasing trend is noticable for both phases as

increase. Physically, fluid begins to behave like a rigid body for bigger value of $A$ since the plastic dynamic viscosity of the fluid $\left(\mu_{B}\right)$ is also higher at which there are high tendency of flow to decelerate. Fig. 4 and Fig. 5 illustrate the effects of magnetic field, $M$ and aligned angle, $\alpha_{1}$ on the velocity profile respectively. It is noticed from both

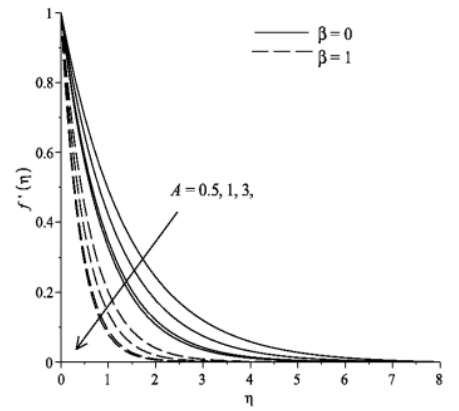

Fig. 2 Influence of $A$ for velocity profile with and without dust particle.

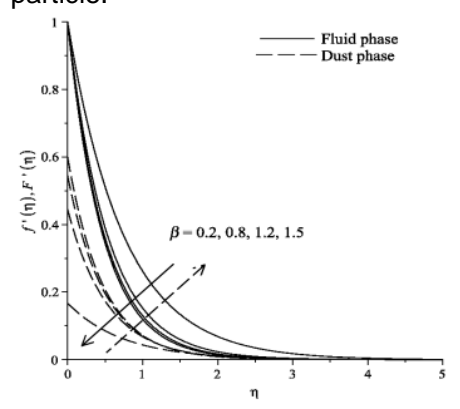

Fig. 6 Influence of $\beta$ on velocity profile.

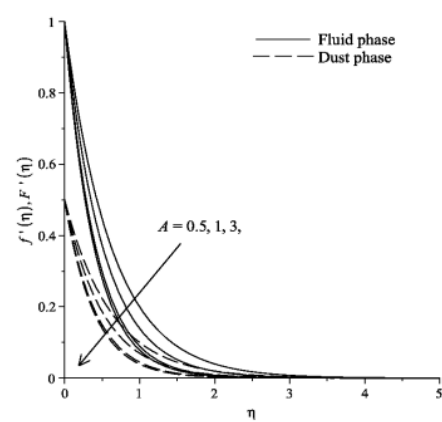

Fig. 3 Influence of $A$ on velocity profile.

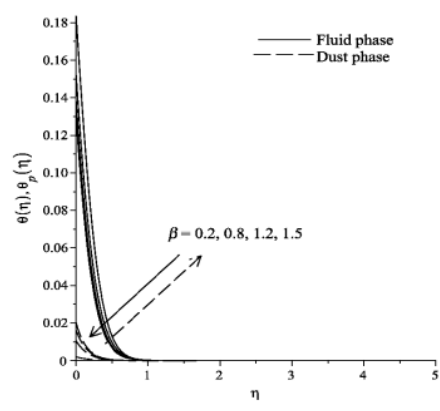

Fig. 7 Influence of $\beta$ on temperature profile.

figures that the increasing $M$ and $\alpha_{1}$ reduces the velocity profile for fluid and dust phases. The increasing aligned angle enhances the development of magnetic field which in turns generate the Lorentz force that act opposite to the flow direction and slower the mobility of the fluid flow. One of the most significant findings to emerge from this study is that the aligned angle determines the position of magnetic field effect as $\alpha_{1}$ can be varies from $0^{\circ}-90^{\circ}$ and if $\alpha_{1}=0$ reflect to the absence of magnetic field effect. Meanwhile, at $\alpha_{1}=\pi / 2$ the present problem is identical to the transverse magnetic field flow. The velocity of fluid literally causes the dust phase velocity, one can observed that both phases decreases with increasing value of $M$ and $\alpha_{1}$.

Fig. 6 and Fig. 7 demonstrate the effect of fluid-particle interaction parameter, $\beta$ on the velocity and temperature profiles for both phases. The decreasing trend is observed for fluid phase and dust phase showed increasing trend as $\beta$ increases. Theoretically, increasing in $\beta$ reduces the relaxation time of particle velocity and enhances the drag force (Stokes's law). In the physical of view, for a large value of $\beta$ causes the growth of the drag force generated by dust particle that behaves opposite to the moving fluid. Actually, this forces continously decrease the velocity of fluid so that both velocities of dust and fluid phase become equlibrium. The dust particle is the source of absorbing the heat from the fluid, thus the decreasing temperature profile of fluid phase is expected.

Fig. 8 and Fig. 9 captured the effects of conjugate parameter, $\gamma$ and Prandtl number, Pr on temperature profile for both phases. A rise in $\gamma$ increases the heat transfer strength which led to enhance the capability to transfer the heat which resulted to the increases in temperature of fluid. Meanwhile, increasing on values of Pr decreases the temperature for both phases due to the fact that larger Pr imply to the lower thermal diffusivity and thus weaken the thermal boundary layer thickness. From the mathematical understanding as denoted by Eqs. (10) - (13), both parameters have no significant effect on flow properties and those parameters can also be used as the control agent in rate of cooling for certain devices.

Table 3 provides the values of skin friction and Nusselt number for different values of magnetic parameter, aligned angle and Casson parameter. The increasing magnitude of skin friction coefficient and decreasing of Nusselt number has been perceived in increasing those parameters. The results presented in Table 3 is reserved for the reference for future study.

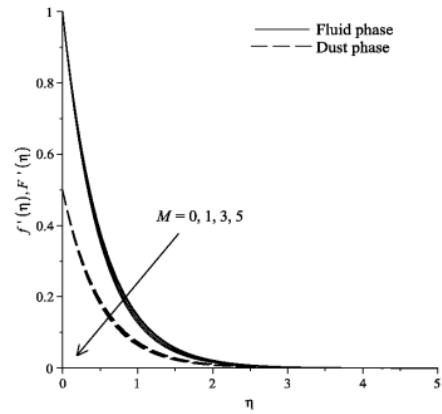

Fig. 4 Influence of $M$ on velocity profile.

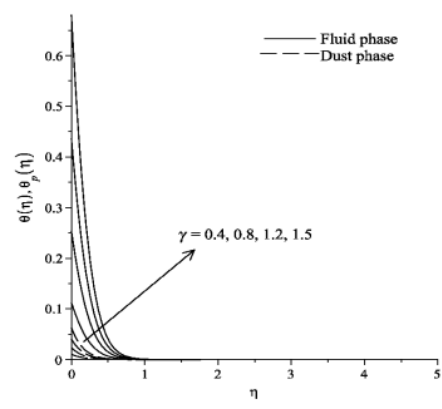

Fig. 8 Influence of $\gamma$ on temperature profile

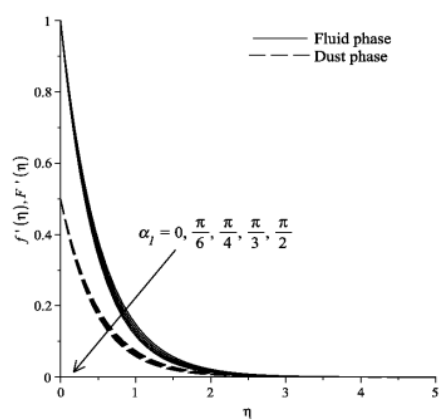

Fig. 5 Influence of $\alpha_{1}$ on velocity profile.

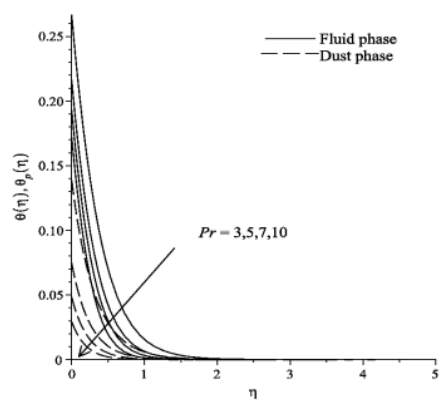

Fig. 9 Influence of $\operatorname{Pr}$ on temperature profile. 
Table 1 Comparision results for $f^{\prime \prime}(0)$, when $\beta=N=0, A, \lambda \rightarrow \infty$.

\begin{tabular}{ccc}
\hline $\boldsymbol{M}$ & (Andersson et al., 1992) & Present \\
\hline 0.0 & -1.0000 & -1.0000 \\
0.2 & -1.0954 & -1.0954 \\
0.5 & -1.2247 & -1.2247 \\
1.0 & -1.4142 & -1.4141 \\
1.2 & -1.4832 & -1.4832 \\
1.5 & -1.5811 & -1.5811 \\
2.0 & -1.7321 & -1.7320 \\
\hline
\end{tabular}

Table 2 Comparision results for $\theta(0)$, when $M=\beta=N=0, \lambda \rightarrow \infty$ and $\gamma=1$.

\begin{tabular}{cccc}
\hline Pr & (Salleh et al., 2010) & Exact Eq. (15) & Present \\
\hline 3 & 6.0258 & 6.0516 & 6.0513 \\
5 & 1.7659 & 1.7604 & 1.7604 \\
7 & 1.1351 & 1.1168 & 1.1168 \\
10 & 0.7653 & 0.7645 & 0.7645 \\
100 & 0.1612 & 0.14781 & 0.1478 \\
\hline
\end{tabular}

Table 3 Computation of $f^{\prime \prime}(0)$ and $N u_{x} \mathrm{Re}_{x}^{-1 / 2}$ for various values of $M, \alpha_{1}$ and $A$.

\begin{tabular}{ccccc}
\hline $\boldsymbol{M}$ & $\boldsymbol{\alpha}_{1}$ & $\boldsymbol{A}$ & $\boldsymbol{f}^{\prime \prime}(\mathbf{0})$ & $\boldsymbol{N \boldsymbol { u } _ { x }} \mathbf{R e}_{x}^{-1 / 2}$ \\
\hline 0.5 & $\pi / 6$ & 1 & -1.75006 & 2.64540 \\
1 & $\pi / 6$ & 1 & -1.76781 & 2.64037 \\
1.5 & $\pi / 6$ & 1 & -1.78539 & 2.63541 \\
2 & $\pi / 6$ & 1 & -1.80280 & 2.63050 \\
2 & $\pi / 6$ & 1 & -1.80280 & 2.63050 \\
2 & $\pi / 4$ & 1 & -1.87084 & 2.61145 \\
2 & $\pi / 3$ & 1 & -1.93650 & 2.59325 \\
2 & $\pi / 2$ & 1 & -2.00000 & 2.57582 \\
2 & $\pi / 6$ & 1 & -1.80280 & 2.63050 \\
2 & $\pi / 6$ & 3 & -2.20794 & 2.51980 \\
2 & $\pi / 6$ & 6 & -2.36039 & 2.47975 \\
2 & $\pi / 6$ & 10 & -2.43087 & 2.46151 \\
\hline
\end{tabular}

\section{CONCLUSION}

The present study was investigate to determine the effect of dust particle suspension in a fluid. The pigmentation and accumulation of dust particle may obstruct the performance of the certain machine and the numerical analyses might help the engineers to overcome this problem. The velocity of fluid and dust phase is decrease with increasing magnetic field parameter, Casson parameter and aligned angle. For fluid phase, the velocity and temperature is decreased in the increasing of fluid particle interaction parameter. In contrary, the opposite trend is observed for dust phase. The influence of increasing the values of magnetic field parameter, aligned angle and Casson parameter are to increase the magnitude of skin friction and decrease the Nusselt number. The bigger on skin friction coefficient produces more drag force along the sheet and become one of the factors that influence the wall design of certain device.

\section{ACKNOWLEDGEMENT}

The authors gratefully acknowledge the financial support received from Universiti Malaysia Pahang for (RDU 160330, RDU 141306, RDU 170328 and PGRS 170328).

\section{REFERENCES}

Andersson, H., Bech, K., and Dandapat, B. (1992). Magnetohydrodynamic flow of a power-law fluid over a stretching sheet. International Journal of NonLinear Mechanics, 27(6), 929-936.

Aurangzaib, K. A., Mohammad, N., and Sharidan, S. (2013). Unsteady MHD mixed convection flow with heat and mass transfer over a vertical plate in a micropolar fluid-saturated porous medium. Journal of Applied Science and Engineering, 16(2), 141-150.

Chakrabarti, K. (1974). Note on boundary layer in a dusty gas. AIAA Journal, 12(8), 1136-1137.

Datta, N., and Mishra, S. (1982). Boundary layer flow of a dusty fluid over a semi-infinite flat plate. Acta Mechanica, 42(1), 71-83.

Hakeem, A. A., Renuka, P., Ganesh, N. V., Kalaivanan, R., and Ganga, B. (2016). Influence of inclined Lorentz forces on boundary layer flow of Casson fluid over an impermeable stretching sheet with heat transfer. Journal of Magnetism and Magnetic Materials, 401, 354-361.

Isa, S. M., Ali, A., and Shafie, S. (2016). Stagnation point flow of MHD dusty fluid toward stretching sheet with convective surface. Jurnal Teknologi, 78 (3-2), 95-100.

Kalaivanan, R., Renuka, P., Ganesh, N. V., Hakeem, A. K. A., Ganga, B., and Saranya, S. (2015). Effects of Aligned Magnetic Field on Slip Flow of Casson Fluid over a Stretching Sheet. Procedia Engineering, 127, 531538.

Mukhopadhyay, S., De, P. R., Bhattacharyya, K., and Layek, G. (2013). Casson fluid flow over an unsteady stretching surface. Ain Shams Engineering Journal, 4(4), 933-938.

Arifin, N. S., Zokri, S., Kasim, A. R. M., Salleh, M. Z., and Mohammad, N. F. (2016). Numerical Solutions of the Aligned Magnetic Field on the Boundary Layer Flow and Heat Transfer over a Stretching Sheet by using Keller-box Method. The National Conference for Postgraduate Research 2016, 266-274.

Ramesh, G. K., Gireesha, B. J., and Gorla, R. S. R. (2015). Boundary layer flow past a stretching sheet with fluid-particle suspension and convective boundary condition. Heat and Mass Transfer, 51(8), 1061-1066.

Saffman, P.G. (1962). On the stability of laminar flow of a dusty gas. Journal of fluid mechanics, 13(1), 120-128.

Salleh, M., Nazar, R., and Pop, I. (2010). Boundary layer flow and heat transfer over a stretching sheet with Newtonian heating. Journal of the Taiwan Institute of Chemical Engineers, 41(6), 651-655.

Sandeep, N., Sulochana, C., and Kumar, B. R. (2015). Flow and heat transfer in MHD dusty nanofluid past a stretching/shrinking surface with nonuniform heat source/sink. Walailak Journal of Science and Technology (WJST), 14(2), 117-140.

Siddiqa, S., Hossain, M. A., and Saha, S. C. (2015). Two-phase natural convection flow of a dusty fluid. International Journal of Numerical Methods for Heat \& Fluid Flow, 25(7), 1542-1556.

Vajravelu, K., Prasad, K. V., and Datti, P. S. (2013). Hydromagnetic fluid flow and heat transfer at a stretching sheet with fluid-particle suspension and variable fluid properties. Journal of Fluids Engineering, 135(1), 011101. 